Article

\title{
Lie Symmetry Analysis, Exact Solutions, Conservation Laws and Bäcklund Transformations of the Gibbons-Tsarev Equation
}

\author{
Huanhuan Lu and Yufeng Zhang * \\ School of Mathematics, China University of Mining and Technology, Xuzhou 221116, China; \\ TS19080020A31@cumt.edu.cn \\ * Correspondence: zyfxz@cumt.edu.cn; Tel.: +1-362-616-6486
}

Received: 3 August 2020; Accepted: 17 August 2020; Published: 18 August 2020

check for updates

\begin{abstract}
In this paper, we mainly put the Lie symmetry analysis method on the Gibbons-Tsarev equation (GTe) to obtain some new results, including some Lie symmetries, one-parameter transformation groups, explicit invariant solutions in the form of power series. Subsequently, the self-adjointness of the GTe is singled out. It follows that the conservation laws associated with symmetries of GTe are constructed with the aid of Ibragimov' method. Finally, we present the Bäcklund transformations so that more abundant solutions can be worked out.
\end{abstract}

Keywords: symmetry; exact solutions; conservation laws; Bäcklund transformation

\section{Introduction}

During the last decades, searching for the symmetries of the nonlinear differential equations has attracted increasing attention from researchers for their wide applications in investigating the integrability of the original equation. In early reference documentations, various powerful approaches were put forward, one after another, to obtain the symmetries of equations [1-5]. Among them, the Lie symmetry analysis method proposed by Olver is regarded as the most classic and verified to be extremely effective for studying Lie symmetry and related group transformations, conservation laws and invariant solutions [1]. With the aid of the classical Lie symmetry analysis method, a large number of differential equations have been discussed, such as the new fifth-order nonlinear integrable equation [6], the Heisenberg equation [7] and some others [8-11]. However, it is worth noting that the method has not been applied to the Gibbons-Tsurev equation

$$
u_{t t}=u_{x} u_{t x}-u_{t} u_{x x}+1,
$$

which was introduced in Reference [12] to classify finite reductions of the infinite Benney system. In Reference [13], the differential constraints compatible with the Gibbons-Tsarev equation are constructed by generalizing the classical determining equations. Besides that, H.Baran, et al. constructed an infinite series of conservation laws and the algebra of nonlocal symmetries in the covering associated with these conservation laws of GTe by means of the known lax pair [14]. It is well known that exact solutions of nonlinear partial differential equations have played an essential role in the study of many complex physical phenomena and other nonlinear engineering problems. Those leading to constructing exact solutions to nonlinear evolution equations are significant. The investigation of these exact solutions involves a variety of powerful methods, among which the Lie symmetry analysis method is a very effective one. Thus, it is very necessary to investigate the solutions of the Gibbons-Tsurev equation by employing the Lie symmetry analysis method. 
In this article, we aim to calculate the Lie point symmetry and invariant solutions of the Gibbons-Tsarev equation in Section 2. The symmetry generator can be directly derived with the aid of Maple software. It follows that the corresponding one-parameter transformation groups also can be obtained. That means the relation between old solutions and new solutions can also be clearly presented. Subsequently, via the characteristic equation we discuss the group-invariant solutions of Equation (1). The series of solutions can be further generated by taking a particular objective function. In Section 3, we are committed to proving that Equation (1) is nonlinearly self-adjoint according to the related theorem introduced in Reference [15]. Based on that, the new conservation laws can also be constructed by utilizing the new conservation theorem proposed by Ibragimov in Reference [16]. Not only that, there are many other approaches to constructing conservation laws that have been put forward by scholars in succession, including the multiplier method, Noether' method, direct method and some others [17-19]. The Noether' method is not applicable to all differential equations due to the fact that the Noether theorem requires existence of a lagrangian and lagrangian exists only for some special types of differential equations. Compared with Noether's approach, the Ibragimov's method shows greater superiority in constructing conservation laws because the existence of lagrangian is not required by the new theorem. Moreover, the new conservation theorem is based on the concept of an adjoint equation for the original equation and the precondition that the differential equation is nonlinearly self-adjoint must be guaranteed before constructing conservation laws. The Bäcklund transformations of the the Gibbons-Tsarev equation in the reduced form are investigated in terms of the Tu scheme in Section 4. Finally, some conclusions and a summary are given in the last section.

\section{Lie Point Symmetries and Related Invariant Solutions}

In this section, we consider employing the classical Lie symmetry analysis method to study the Lie point symmetries and group-invariant solutions associated with symmetries of the Gibbons-Tsarev equation. We first assume a vector field has the following infinitesimal generator

$$
V=\xi(x, t, u) \frac{\partial}{\partial x}+\eta(x, t, u) \frac{\partial}{\partial t}+\tau(x, t, u) \frac{\partial}{\partial u}
$$

and its second prolongation $\mathrm{pr}^{(2)} V$ can be expressed as

$$
p r^{(2)} V=V+\phi^{t} \frac{\partial}{\partial u_{t}}+\phi^{x} \frac{\partial}{\partial u_{x}}+\phi^{x x} \frac{\partial}{\partial u_{x x}}+\phi^{t t} \frac{\partial}{\partial u_{t t}}+\phi^{t x} \frac{\partial}{\partial u_{t x}},
$$

where

$$
\begin{aligned}
& \phi^{x}=D_{x}\left(\tau-\xi u_{x}-\eta u_{t}\right)+\xi u_{x x}+\eta u_{x t}, \\
& \phi^{t}=D_{t}\left(\tau-\xi u_{x}-\eta u_{t}\right)+\xi u_{x t}+\eta u_{t t}, \\
& \phi^{t x}=D_{t} D_{x}\left(\tau-\xi u_{x}-\eta u_{t}\right)+\xi u_{x x t}+\eta u_{t t x}, \\
& \phi^{t t}=D_{t}^{2}\left(\tau-\xi u_{x}-\eta u_{t}\right)+\xi u_{x t t}+\eta u_{t t t}, \\
& \phi^{x x}=D_{x}^{2}\left(\tau-\xi u_{x}-\eta u_{t}\right)+\xi u_{x x x}+\eta u_{x x t}
\end{aligned}
$$

and the total derivative operator $D_{i}$ is defined by

$$
D_{i}=\frac{\partial}{\partial x^{i}}+u_{i} \frac{\partial}{\partial u}+u_{i j} \frac{\partial}{\partial u_{j}}+\cdots, \quad i=1,2, \quad\left(x^{1}, x^{2}\right)=(t, x) .
$$

As a matter of fact, the Lie point symmetry is obtained if Equation (1) satisfies the following invariance condition

$$
\left.\operatorname{pr}^{(2)} V\left(\triangle_{1}\right)\right|_{\triangle_{1}=0}=0,
$$

here $\triangle_{1}=u_{t t}-u_{x} u_{t x}+u_{t} u_{x x}-1$. It follows that

$$
\phi^{t t}-\phi^{x} u_{t x}-\phi^{t x} u_{x}-\phi^{t} u_{x x}-\phi^{x x} u_{t}=0 .
$$


Substituting Equation (2) into Equation (3) and equating the coefficients of the polynomials to zero yields a system of equations with respect to $\xi, \eta, \tau$. Solving these algebraic equations, one has

$$
\xi=-\frac{1}{2} c_{3} t+\frac{3}{2} c_{1} x+c_{5}, \quad \eta=c_{1} t+c_{2}, \quad \tau=2 c_{1} u+c_{3} x+c_{4} .
$$

Thus, we obtain the following vector fields

$$
V_{1}=\frac{3}{2} x \frac{\partial}{\partial x}+t \frac{\partial}{\partial t}+2 u \frac{\partial}{\partial u}, \quad V_{2}=\frac{\partial}{\partial t}, \quad V_{3}=-\frac{1}{2} t \frac{\partial}{\partial x}+x \frac{\partial}{\partial u}, \quad V_{4}=\frac{\partial}{\partial u}, \quad V_{5}=\frac{\partial}{\partial x} .
$$

Meanwhile, it is not difficult to find that these vector fields $V_{i}(i=1,2, \cdots, 5)$ form a Lie algebra and the corresponding Lie bracket table is given in Table 1.

Table 1. The commutator table.

\begin{tabular}{cccccc}
\hline$\left[V_{i}, V_{j}\right]$ & $V_{1}$ & $V_{\mathbf{2}}$ & $V_{3}$ & $V_{4}$ & $V_{5}$ \\
\hline$V_{1}$ & 0 & $-V_{2}$ & $-\frac{1}{2} V_{3}$ & $-2 V_{4}$ & $-\frac{3}{2} V_{5}$ \\
$V_{2}$ & $V_{2}$ & 0 & $-\frac{1}{2} V_{5}$ & 0 & 0 \\
$V_{3}$ & $\frac{1}{2} V_{3}$ & $\frac{1}{2} V_{5}$ & 0 & 0 & $-V_{4}$ \\
$V_{4}$ & $2 V_{4}$ & 0 & 0 & 0 & 0 \\
$V_{5}$ & $\frac{3}{2} V_{5}$ & 0 & $V_{4}$ & 0 & 0 \\
\hline
\end{tabular}

In order to investigate the invariant solutions for Equation (1), it is very important to search for Lie group transformations which can be generated by solving the following initial problems

$$
\begin{array}{ll}
\frac{d \tilde{x}(\epsilon)}{d \epsilon}=\xi(\tilde{x}(\epsilon), \tilde{t}(\epsilon), \tilde{u}(\epsilon)), & \tilde{x}(0)=x, \\
\frac{d \tilde{t}(\epsilon)}{d \epsilon}=\eta(\tilde{x}(\epsilon), \tilde{t}(\epsilon), \tilde{u}(\epsilon)), & \tilde{t}(0)=t, \\
\frac{d \tilde{u}(\epsilon)}{d \epsilon}=\tau(\tilde{x}(\epsilon), \tilde{t}(\epsilon), \tilde{u}(\epsilon)), & \tilde{u}(0)=u,
\end{array}
$$

where $\epsilon$ is a parameter. Therefore, the one-parameter Lie symmetry group generated by the infinitesimal generator $V_{i}(i=1,2, \cdots, 5)$ can be expressed as below, respectively.

$$
\begin{aligned}
& g_{1}(x, t, u)=e^{V_{1}}(x, t, u)=\left(e^{\frac{3}{2} \epsilon} x, e^{\epsilon} t, e^{2 \epsilon} u\right), \\
& g_{2}(x, t, u)=e^{V_{2}}(x, t, u)=(x, \epsilon+t, u), \\
& g_{3}(x, t, u)=e^{V_{3}}(x, t, u)=\left(-\frac{1}{2} t \epsilon+x, t, \epsilon x+u\right), \\
& g_{4}(x, t, u)=e^{V_{4}}(x, t, u)=(x, t, u+\epsilon), \\
& g_{5}(x, t, u)=e^{V_{5}}(x, t, u)=(x+\epsilon, t, u) .
\end{aligned}
$$

If we denote the action of the above symmetry group generated by $V_{i}$ on a solution $f(x, t)$ to Equation (1) by $g_{i}(\epsilon) f(x, t)$, we can get the corresponding new solutions:

$$
\begin{aligned}
& g_{1}(\epsilon) f(x, t)=e^{-2 \epsilon} f\left(e^{\frac{3}{2} \epsilon} x, e^{\epsilon} t\right), \\
& g_{2}(\epsilon) f(x, t)=f(x, t-\epsilon), \\
& g_{3}(\epsilon) f(x, t)=f\left(-\frac{1}{2} t \epsilon+x, t\right)-\epsilon x, \\
& g_{4}(\epsilon) f(x, t)=f(x, t)-\epsilon, \\
& g_{5}(\epsilon) f(x, t)=f(x-\epsilon, t) .
\end{aligned}
$$


In what follows, we seek invariant solutions of GTe with the aid of characteristic equations. For the vector field $V_{1}$, we have the following characteristic equation

$$
\frac{d x}{\frac{3}{2} x}=\frac{d t}{t}=\frac{d u}{2 u}
$$

which has the group-invariant solution

$$
u=t^{2} g(\xi), \quad \xi=x^{\frac{2}{3}} t^{-1}
$$

Substituting Equation (5) into Equation (1) gives rise to

$$
2 g(\xi)-2 g^{\prime}(\xi)+\xi^{2} g^{\prime \prime}(\xi)-\frac{2}{9} \xi^{-1} g^{\prime 2}(\xi)+\frac{8}{9} \xi^{-1} g(\xi) g^{\prime \prime}(\xi)-\frac{4}{9} \xi^{-2} g(\xi) g^{\prime}(\xi)-1=0
$$

In order to construct power series solution, we assume $g(\xi)$ has the following form

$$
g(\xi)=\sum_{n=0}^{\infty} c_{n} \xi^{n}
$$

Substituting Equation (7) into Equation (6) and equating the coefficients of $\xi^{n}(n \geq 0)$ to zero, one has

$$
\begin{aligned}
& \xi^{0}:-\frac{4}{9} c_{0} c_{1}=0 \\
& \xi^{1}:-\frac{2}{3} c_{1}^{2}+\frac{8}{9} c_{0} c_{2}=0 \\
& \xi^{2}: 2 c_{0}-\frac{8}{9} c_{1} c_{2}+4 c_{0} c_{3}-1=0 \\
& \xi^{3}: 2 c_{1}+\frac{20}{9} c_{1} c_{3}+\frac{80}{9} c_{0} c_{4}=0 \\
& \xi^{4}: 3 c_{1} c_{4}+c_{2} c_{3}+7 c_{0} c_{5}=0 \\
& \xi^{5}: 9 c_{3}+58 c_{1} c_{5}+36 c_{2} c_{4}+27 c_{3}^{2}+108 c_{0} c_{6}=0 \\
& \cdots
\end{aligned}
$$

and solving the above equations, we get

$$
c_{0} \neq 0, c_{1}=0, c_{2}=0, c_{3}=\frac{1-2 c_{0}}{4 c_{0}}, c_{4}=0, c_{5}=0, c_{6}=\frac{-c_{3}-3 c_{3}^{2}}{12 c_{0}}=\frac{-4 c_{0}^{2}+8 c_{0}-3}{192 c_{0}^{3}}, \cdots
$$

Thus, the explicit form of $g(\xi)$ can be written as

$$
g(\xi)=c_{0}+\frac{1-2 c_{0}}{4 c_{0}} \xi^{3}+\frac{-4 c_{0}^{2}+8 c_{0}-3}{192 c_{0}^{3}} \xi^{6}+\cdots
$$

where $c_{0}$ is an arbitrary constant. Hence, we acquire the following analytic power series solution

$$
u(x, t)=t^{2}\left(c_{0}+\frac{1-2 c_{0}}{4 c_{0}} \xi^{3}+\frac{-4 c_{0}^{2}+8 c_{0}-3}{192 c_{0}^{3}} \xi^{6}+\cdots\right) .
$$

In terms of Equation (4), we can also introduce the following group-invariant solution

$$
u=x^{\frac{4}{3}} f(\xi), \quad \xi=x^{\frac{2}{3}} t^{-1} .
$$


Substituting Equation (8) into Equation (1) yields

$$
2 \xi^{3} f^{\prime}(\xi)+\xi^{4} f^{\prime \prime}(\xi)+\frac{20}{9} \xi^{2} f(\xi) f^{\prime}(\xi)+\frac{8}{9} \xi^{3} f(\xi) f^{\prime \prime}(\xi)-\frac{2}{9} \xi^{3} f^{\prime 2}(\xi)-1=0 .
$$

Similar to the above discussion, we still suppose $f(\xi)$ is of the form $f(\xi)=\sum_{n=0}^{\infty} c_{n} \xi^{n}$. Thus Equation (9) can be transformed into

$$
\begin{aligned}
& 2 \sum_{n=0}^{\infty}(n+1) c_{n+1} \xi^{n+3}+\sum_{n=0}^{\infty}(n+1)(n+2) c_{n+2} \xi^{n+4}+\frac{20}{9} c_{0} c_{1} \xi^{2}+\frac{20}{9} \sum_{n=1}^{\infty} \sum_{k=0}^{n}(n+1-k) c_{k} c_{n+1-k} \xi^{n+2}+\frac{16}{9} c_{0} c_{2} \xi^{3} \\
& +\frac{8}{9} \sum_{n=1}^{\infty} \sum_{k=0}^{n}(n+2-k)(n+1-k) c_{k} c_{n+2-k} \xi^{n+3}-\frac{2}{9} c_{1}^{2} \xi^{3}-\frac{2}{9} \sum_{n=1}^{\infty} \sum_{k=1}^{n} k(n+2-k) c_{k} c_{n+2-k} \xi^{n+3}-1=0 .
\end{aligned}
$$

Obviously, Equation (10) has no solution because of the existence of the single term 1.

For $V_{3}$, one has the characteristic equation

$$
\frac{d x}{-\frac{1}{2} t}=\frac{d u}{x}=\frac{d t}{0}
$$

Similarly, we introduce the following group-invariant solution

$$
u=f(\xi)-\frac{x^{2}}{t}, \quad \xi=t
$$

And inserting Equation (11) into Equation (1), we get

$$
f^{\prime \prime}(\xi)-2 \frac{f^{\prime}(\xi)}{\xi}-1=0,
$$

that is equivalent to

$$
\xi f^{\prime \prime}(\xi)-2 f^{\prime}(\xi)-\xi=0
$$

Substituting $\xi f^{\prime \prime}(\xi)=f^{\prime}(\xi)+\xi^{2}\left(\frac{f^{\prime}(\xi)}{\xi}\right)^{\prime}$ into Equation (12) gives

$$
\left(\frac{f^{\prime}(\xi)}{\xi}\right)^{\prime}-\frac{1}{\xi} \frac{f^{\prime}(\xi)}{\xi}-\frac{1}{\xi}=0
$$

Setting $\frac{f^{\prime}(\tilde{\xi})}{\tilde{\xi}}=y(\xi)$, then Equation (13) becomes

$$
y^{\prime}-\frac{1}{\xi} y=\frac{1}{\xi^{\prime}}
$$

whose solution reads

$$
y=e^{\int \frac{1}{\xi} d \xi}\left(\int \frac{1}{\xi} e^{-\int \frac{1}{\xi} d \xi} d \xi+c_{1}\right)=c_{1} \xi-1 .
$$

Inserting $\frac{f^{\prime}(\xi)}{\xi}=y(\xi)$ into Equation (15) and integrating once with respect to $\xi$ produces

$$
f(\xi)=\frac{c_{1}}{3} \xi^{3}-\frac{1}{2} \xi^{2}+c_{2}
$$

where $c_{1}, c_{2}$ are arbitrary integration constants. 
Hence, we have the following invariant solutions

$$
u(x, t)=\frac{c_{1}}{3} \xi^{3}-\frac{1}{2} \xi^{2}+c_{2}-\frac{x^{2}}{t} .
$$

\section{Nonlinear Self-Adjointness and Conservation Laws}

Since Ibragimov proposed a direct and effective method for constructing conservation laws, which overcomes the shortcomings of previous methods, more and more experts and scholars have devoted themselves to utilizing this method to construct conservation laws associated with symmetries of nonlinearly self-adjoint equations [16]. After that, the approach for constructing conserved vectors of fractional differential equations also has been put forward and applied in References [20,21] by using the explicit Formula (40) shown in Reference [22]. It is worth emphasizing that we must prove that a given differential equation is nonlinearly self-adjoint before constructing corresponding conservation laws. So in this section, we first focus on proving Equation (1) is nonlinearly self-adjoint. Before that, let us give some fundamental definitions and notations associated with nonlinear self-adjointness and conservation laws [15].

Let us consider a system of $m$ differential equations

$$
F_{\alpha}\left(x, u, u_{(1)}, u_{(2)}, \cdots, u_{(s)}\right)=0, \quad \alpha=1,2, \cdots, m,
$$

with $m$ dependent variables $u=\left(u^{1}, \cdots, u^{m}\right)$ and $n$ independent variables $x=\left(x^{1}, \cdots, x^{n}\right)$.

Definition 1. The adjoint equations to Equation (18) are given by

$$
F_{\alpha}^{*}\left(x, u, v, u_{(1)}, v_{(1)}, \cdots, u_{(s)}, v_{(s)}\right)=0, \quad \alpha=1,2, \cdots, m,
$$

with

$$
F_{\alpha}^{*}\left(x, u, v, u_{(1)}, v_{(1)}, \cdots, u_{(s)}, v_{(s)}\right)=\frac{\delta \mathcal{L}}{\delta u^{\alpha}},
$$

where $\mathcal{L}$ is the formal Lagrangian for Equation (18) defined by

$$
\mathcal{L}=v^{\beta} F_{\beta}=\sum_{\beta=1}^{m} v^{\beta} F_{\beta}
$$

Here $v_{(1)}, \cdots, v_{(s)}$ are the sets of the partial derivatives of new dependent variables $v=\left(v^{1}, \cdots, v^{m}\right)$, the Euler-lagrange operator is

$$
\frac{\delta}{\delta u^{\alpha}}=\frac{\partial}{\partial u^{\alpha}}+\sum_{s=1}^{\infty}(-1)^{s} D_{i 1} \cdots D_{i s} \frac{\partial}{\partial u_{i 1 \ldots i s}^{\alpha}}, \quad \alpha=1, \ldots, m
$$

and the total differentiations $D_{i}$ with two dependent variables $u, v$ are defined by

$$
D_{i}=\frac{\partial}{\partial x^{i}}+u_{i}^{\alpha} \frac{\partial}{\partial u^{\alpha}}+v_{i}^{\alpha} \frac{\partial}{\partial v^{\alpha}}+u_{i j}^{\alpha} \frac{\partial}{\partial u_{j}^{\alpha}}+v_{i j}^{\alpha} \frac{\partial}{\partial v_{j}^{\alpha}}+\cdots
$$

Definition 2. The system Equation (18) is nonlinearly self-adjoint if there exist functions $v^{\alpha}=\varphi^{\alpha}(x, u)(\alpha=1,2, \cdots, m)$ that solve the adjoint system Equation (19) for all solutions $u(x)$ of Equation (18) and satisfy the condition $\varphi^{\alpha}(x, u) \neq 0$.

In addition, we have the following equivalent definition 
Definition 3. The system Equation (18) is nonlinearly self-adjoint if there exist a substitution $v=\varphi(x, u) \neq 0$ such that the equation

$$
F_{\alpha}^{*}\left(x, u, \varphi, u_{(1)}, \varphi_{(1)}, \cdots, u_{(s)}, \varphi_{(s)}\right)=\lambda_{\alpha} F_{\alpha}\left(x, u, u_{(1)}, \cdots, u_{(s)}\right)
$$

is satisfied identically in the variables, $x, u, u_{(1)}, \cdots, u_{(s)}$, where $\lambda_{\alpha}=\lambda_{\alpha}\left(x, u, u_{(1)}, \cdots, u_{(s)}\right)$ is an undetermined variable coefficients.

Theorem 1. Let the system Equation (18) be nonlinearly self-adjoint. The any Lie point, contact or Lie-Bäcklund symmetry

$$
X=\xi^{i}\left(x, u, u_{x}, \cdots\right) \frac{\partial}{\partial x^{i}}+\eta^{\alpha}\left(x, u, u_{x}, \cdots\right) \frac{\partial}{\partial u^{\alpha}}
$$

leads to conservation laws

$$
\left.D_{i}\left(C^{i}\right)\right|_{F_{\alpha}=0}=0,
$$

where

$$
\begin{gathered}
C^{i}=W^{\alpha}\left[\frac{\partial L}{\partial u_{i}^{\alpha}}-D_{j}\left(\frac{\partial L}{\partial u_{i j}^{\alpha}}\right)+D_{j} D_{k}\left(\frac{\partial L}{\partial u_{i j k}^{\alpha}}\right)-\cdots\right]+D_{j}\left(W^{\alpha}\right)\left[\frac{\partial L}{\partial u_{i j}^{\alpha}}-D_{k}\left(\frac{\partial L}{\partial u_{i j k}^{\alpha}}\right)+\cdots\right]+D_{j} D_{k}\left(W^{\alpha}\right)\left[\frac{\partial L}{\partial u_{i j k}^{\alpha}}-\cdots\right], \\
W^{\alpha}=\eta^{\alpha}-\xi^{j} u_{j}^{\alpha} .
\end{gathered}
$$

Based on the given definitions and notations, the formal lagrangian for Equation (1) can be written as

$$
\mathcal{L}=v\left(u_{t t}-u_{x} u_{t x}+u_{t} u_{x x}-1\right) .
$$

And substituting Equation (21) into Equation (19) gives the following adjoint equation to Equation (1):

$$
\begin{aligned}
F^{*} & =D_{t}^{2}(v)+D_{x}\left(v u_{x t}\right)-D_{x} D_{t}\left(u_{x} v\right)+D_{x}^{2}\left(u_{t} v\right)-D_{t}\left(v u_{x x}\right) \\
& =v_{t t}-2 u_{x x} v_{t}-u_{x} v_{x t}+2 u_{x t} v_{x}+u_{t} v_{x x}=0 .
\end{aligned}
$$

According to Definition 3, the nonlinear self-adjointness condition for Equation (1) has the following form

$$
v_{t t}-2 u_{x x} v_{t}-u_{x} v_{x t}+2 u_{x t} v_{x}+u_{t} v_{x x}=\lambda\left(u_{t t}-u_{x} u_{t x}+u_{t} u_{x x}-1\right),
$$

where $\lambda=\lambda(x, t, u)$. Denoting $v=\varphi(x, t, u)$, and substituting into Equation (23) yields

$$
\begin{aligned}
& \varphi_{t t}+\varphi_{t u} u_{t}+\varphi_{u} u_{t t}+\varphi_{u u} u_{t}^{2}+\varphi_{t u} u_{t}-\varphi_{t} u_{x x}-\varphi_{x t} u_{x}-\varphi_{t u} u_{x}^{2}+2 \varphi_{x} u_{x t}+\varphi_{u} u_{x} u_{x t}+\varphi_{x x} u_{t} \\
& +\varphi_{u x} u_{x} u_{t}-\varphi_{t} u_{x x}-\varphi_{u} u_{t} u_{x x}=\lambda\left(u_{t t}-u_{x} u_{t x}+u_{t} u_{x x}-1\right) .
\end{aligned}
$$

Equating the coefficients for the different derivative terms of $u(x, t)$ and their product terms to zero yields

$$
\varphi_{t}=0, \quad \varphi_{x}=0, \quad \varphi_{u}=0,
$$

which indicates $\varphi=$ constant $\neq 0$. Thus, Equation (1) is nonlinearly self-adjoint. It follows that we can obtain some conservation laws of Equation (1) according to Theorem 1 .

For the generator $V_{1}=\frac{3}{2} x \frac{\partial}{\partial x}+t \frac{\partial}{\partial t}+2 u \frac{\partial}{\partial u}$, we have $W=2 u-\frac{3}{2} x u_{x}-t u_{t}$. Thus, formulas Equation (20) yield the following conserved vector

$$
\begin{aligned}
C^{1} & =W\left[\frac{\partial \mathcal{L}}{\partial u_{t}}-D_{x}\left(\frac{\partial \mathcal{L}}{\partial u_{x t}}\right)\right]+D_{x}(W)\left[\frac{\partial \mathcal{L}}{\partial u_{x t}}\right]+D_{t}(W)\left[\frac{\partial \mathcal{L}}{\partial u_{t t}}\right]-D_{t}\left(\frac{\partial \mathcal{L}}{\partial u_{t t}}\right) \\
& =4 u v u_{x x}+2 u u_{x} v_{x}-2 u v_{t}-3 x v u_{x} u_{x x}-\frac{3}{2} x u_{x}^{2} v_{x}+\frac{3}{2} x u_{t} v_{t}-2 t u_{t} v u_{x x}-t u_{x} u_{t} v_{x}+t u_{t} v_{t}-\frac{1}{2} u_{x}^{2} v \\
& +\frac{3}{2} x u_{x} u_{x x} v+t u_{t x} u_{x} v+v u_{t}-\frac{3}{2} x u_{x t} v-t v u_{t t}
\end{aligned}
$$




$$
\begin{aligned}
C^{2} & =W\left[\frac{\partial \mathcal{L}}{\partial u_{x}}-D_{x}\left(\frac{\partial \mathcal{L}}{\partial u_{x x}}\right)-D_{t}\left(\frac{\partial \mathcal{L}}{\partial u_{x t}}\right)\right]+D_{t}(W)\left[\frac{\partial \mathcal{L}}{\partial u_{x t}}\right]+D_{x}(W)\left[\frac{\partial \mathcal{L}}{\partial u_{x x}}\right] \\
& =-2 u v u_{x t}-2 u u_{t} v_{x}+2 u u_{x} v_{t}+\frac{3}{2} x u_{x} v u_{t x}+\frac{3}{2} x u_{t} u_{x} v_{x}-\frac{3}{2} x u_{x}^{2} v_{t}+t v u_{t} u_{t x}+t u_{t}^{2} v_{x}-t u_{t} u_{x} v_{t}-u_{t} u_{x} v \\
& +\frac{3}{2} x u_{x t} u_{x} v+t u_{t t} u_{x} v+\frac{1}{2} u_{x} u_{t} v-\frac{3}{2} x u_{x x} u_{t} v-t u_{x t} u_{t} v .
\end{aligned}
$$

For the generator $V_{2}=\frac{\partial}{\partial t}$, we have $W=-u_{t}$. Thus formulas Equation (20) yield the following conserved vector

$$
\begin{aligned}
& C^{1}=-2 u_{t} v u_{x x}-u_{t} u_{x} v_{x}+u_{t} v_{t}+u_{x t} u_{x} v-u_{t t} v, \\
& C^{2}=u_{t}^{2} v_{x}-u_{t} u_{x} v_{t}+u_{t t} u_{x} v .
\end{aligned}
$$

For the generator $V_{3}=-\frac{1}{2} t \frac{\partial}{\partial x}+x \frac{\partial}{\partial u}$, we have $W=x+\frac{1}{2} t u_{x}$. Thus formulas Equation (20) yield the following conserved vector

$$
\begin{aligned}
& C^{1}=2 x v u_{x x}+x u_{x} v_{x}-x v_{t}+\frac{t}{2} v u_{x} u_{x x}+\frac{1}{2} t u_{x}^{2} v_{x}-\frac{1}{2} t u_{x} v_{t}-u_{x} v+\frac{1}{2} v u_{x}+\frac{1}{2} t u_{x t} v \\
& C^{2}=-x v u_{t x}-x u_{t} v_{x}+x u_{x} v_{t}-t v u_{x} u_{x t}-\frac{1}{2} t u_{x} u_{t} v_{x}+\frac{1}{2} t u_{x}^{2} v_{t}-\frac{1}{2} u_{x}^{2} v+u_{t} v+\frac{1}{2} t u_{x x} u_{t} v .
\end{aligned}
$$

For the generator $V_{4}=\frac{\partial}{\partial u}$, we have $W=1$. Thus formulas Equation (20) yield the following conserved vector

$$
\begin{aligned}
& C^{1}=2 v u_{x x}+u_{x} v_{x}-v_{t} \\
& C^{2}=-v u_{t x}-u_{t} v_{x}+u_{x} v_{t} .
\end{aligned}
$$

For the generator $V_{5}=\frac{\partial}{\partial x}$, we have $W=-u_{x}$. Thus formulas Equation (20) yield the following conserved vector

$$
\begin{aligned}
& C^{1}=-v u_{x x} u_{x}-u_{x}^{2} v_{x}+u_{x} v_{t}-u_{x t} v \\
& C^{2}=2 v u_{x t} u_{x}+u_{x} u_{t} v_{x}-u_{x}^{2} v_{t}-u_{x x} u_{t} v
\end{aligned}
$$

Remark 1. Obviously, it can be clearly seen that the derived conservation laws involve not only the solution $u(x, t)$ of the original equation, but also the solution $v(x, t)$ of the adjoint equation. Hence, what is worth emphasizing is that the above given conservation laws are for Equation (1) and the adjoint equation Equation (22). In order to obtain the conservation laws of the original equation, we must solve $v(x, t)$ from the adjoint equation. To this end, we set $\xi=t+\beta x$ in Equation (22). Thus obtaining

$$
v^{\prime \prime}(\xi)-2 u_{x x} v^{\prime}(\xi)-\beta u_{x} v^{\prime \prime}(\xi)+2 \beta u_{x t} v^{\prime}(\xi)+\beta^{2} u_{t} v^{\prime \prime}(\xi)=0
$$

that is equivalent to

$$
\left(1-\beta u_{x}+\beta^{2} u_{t}\right) v^{\prime \prime}(\xi)+\left(2 \beta u_{x t}-2 u_{x x}\right) v^{\prime}(\xi)=0 .
$$

Letting $v^{\prime}=y(\xi)$, then Equation (24) gives

$$
\frac{d y}{d \xi}=-\frac{2 \beta u_{x t}-2 u_{x x}}{1-\beta u_{x}+\beta^{2} u_{t}} y
$$

whose solution reads

$$
y=e^{\int-\frac{2 \beta u_{x t}-2 u_{x x}}{1-\beta u_{x}+\beta^{2} u_{t}} d \xi}
$$

i.e.,

$$
v=\int e^{\int-\frac{2 \beta u_{x t}-2 u_{x x}}{1-\beta u_{x}+\beta^{2} u_{t}} d \xi} d \xi .
$$

Substituting Equation (25) and $\xi=t+\beta x$ into the derived conservation vectors, we can derive the conservation laws for the original equation Equation (1). 


\section{Bäcklund Transformations}

The main purpose of this section is to find the Bäcklund transformations of the reduced form of Equation (1). For the sake of brevity, we restrict our consideration to the Gibbons-Tsarev equation in the following reduced form

$$
w_{t t}=w_{x} w_{t x}-w_{t} w_{x x}
$$

To begin with, we let $F(w)=w_{x} w_{t x}-w_{t} w_{x x}, \quad u=w+w^{\prime}, \quad v=w-w^{\prime}$, where $w$ and $w^{\prime}$ are solutions to Equation (26), that is

$$
w_{t t}=F(w), \quad w_{t t}^{\prime}=F\left(w^{\prime}\right) .
$$

By adding and subtracting the above two equations, we can get

$$
\begin{aligned}
& u_{t t}=F\left(\frac{u+v}{2}\right)+F\left(\frac{u-v}{2}\right), \\
& v_{t t}=F\left(\frac{u+v}{2}\right)-F\left(\frac{u-v}{2}\right) .
\end{aligned}
$$

Direct calculation yields

$$
\begin{aligned}
& u_{t t}=\frac{u_{x} u_{t x}+v_{x} v_{t x}-u_{t} u_{x x}-v_{t} v_{x x}}{2}, \\
& v_{t t}=\frac{u_{x} v_{t x}+v_{x} u_{t x}-u_{t} v_{x x}-v_{t} u_{x x}}{2} .
\end{aligned}
$$

Based on the selection of the Bäcklund transformation in [23,24], here we suppose the Bäcklund transformation of Equation (1) has the following form

$$
\begin{aligned}
& u_{t}=f\left(u, v, u_{x}, v_{x}\right), \\
& v_{t}=g\left(u, v, u_{x}, v_{x}\right) .
\end{aligned}
$$

Then we have

$$
\begin{gathered}
u_{t t}=f_{u} u_{t}+f_{v} v_{t}+f_{u_{x}} u_{x t}+f_{v_{x}} u_{x t}, \\
u_{x t}=f_{u} u_{x}+f_{v} v_{t}+f_{u_{x}} u_{x x}+f_{v_{x}} v_{x x}, \\
v_{t t}=g_{v} v_{t}+g_{u} u_{t}+g_{v_{x}} v_{x t}+g_{u_{x}} u_{x t}, \\
v_{x t}=g_{v} v_{x}+g_{u} u_{x}+g_{u_{x}} u_{x x}+g_{v_{x}} v_{x x} .
\end{gathered}
$$

Substituting Equations (30a), (30b), (31b) and (31d) into Equation (31a) yields

$$
u_{t t}=f_{u} f+f_{v} g+f_{u_{x}} f_{u} u_{x}+f_{u_{x}} f_{v} v_{x}+f_{u_{x}}^{2} u_{x x}+f_{u_{x}} f_{v_{x}} v_{x x}+f_{v_{x}} g_{v} v_{x}+f_{v_{x}} g_{u} u_{x}+f_{v_{x}} g_{u_{x}} u_{x x}+f_{v_{x}} g_{v_{x}} v_{x x} \text {. }
$$

Substituting Equations (30a), (30b), (31b) and (31d) into Equation (31c) yields $v_{t t}=g_{v} g+g_{u} f+g_{v_{x}} g_{v} v_{x}+g_{v_{x}} g_{u} u_{x}+g_{v_{x}} g_{u_{x}} u_{x x}+g_{v_{x}}^{2} v_{x x}+g_{u_{x}} f_{u} u_{x}+g_{u_{x}} f_{v} v_{x}+g_{u_{x}} f_{u_{x}} u_{x x}+g_{u_{x}} f_{v_{x}} v_{x x}$.

Substituting Equations (30a), (30b), (31b) and (31d) into Equations (29a) and (29b) yields, respectively

$$
\begin{aligned}
& u_{t t}=\frac{f_{u} u_{x}^{2}+f_{v} u_{x} v_{x}+f_{u_{x}} u_{x} u_{x x}+f_{v_{x}} u_{x} v_{x x}+g_{v} v_{x}^{2}+g_{u} u_{x} v_{x}+g_{u_{x}} u_{x x} v_{x}+g_{v_{x}} v_{x} v_{x x}-f u_{x x}-g v_{x x}}{2}, \\
& v_{t t}=\frac{g_{v} u_{x} v_{x}+u_{x}^{2} g_{u}+g_{u_{x}} u_{x} u_{x x}+g_{v_{x}} u_{x} v_{x x}+f_{u} v_{x} u_{x}+f_{v} v_{x}^{2}+f_{u_{x}} u_{x x} v_{x}+f_{v_{x}} v_{x} v_{x x}-f v_{x x}-g u_{x x}}{2} .
\end{aligned}
$$


Setting the coefficients of the same terms on the right of the equations Equations (32) and (34) be equal, one gets

$$
\begin{gathered}
u_{x x}: f_{u_{x}}^{2}+f_{v_{x}} g_{u_{x}}=\frac{1}{2} f_{u_{x}} u_{x}+\frac{1}{2} g_{u_{x}} v_{x}-\frac{1}{2} f, \\
v_{x x}: f_{u_{x}} f_{v_{x}}+f_{v_{x}} g_{v_{x}}=\frac{1}{2} f_{v_{x}} u_{x}+\frac{1}{2} g_{v_{x}} v_{x}-\frac{1}{2} g, \\
u_{x}^{2}: f_{u}=0, \quad v_{x}^{2}: g_{v}=0, \\
u_{x}: f_{u_{x}} f_{u}+f_{v_{x}} g_{u}=\frac{1}{2}\left(f_{v}+g_{u}\right) v_{x}, \quad v_{x}: f_{u_{x}} f_{v}+f_{v_{x}} g_{v}=0, \\
f f_{u}+f_{v} g=0 .
\end{gathered}
$$

For Equations (33) and (35), by carrying out similar procedures as above, one has

$$
\begin{gathered}
u_{x x}: g_{v_{x}} g_{u_{x}}+g_{u_{x}} f_{u_{x}}=\frac{1}{2} g_{u_{x}} u_{x}+\frac{1}{2} f_{u_{x}} v_{x}-\frac{1}{2} g, \\
v_{x x}: g_{v_{x}}^{2}+f_{v_{x}} g_{u_{x}}=\frac{1}{2} g_{v_{x}} u_{x}+\frac{1}{2} f_{v_{x}} v_{x}-\frac{1}{2} f, \\
u_{x}^{2}: g_{u}=0, \quad v_{x}^{2}: f_{v}=0, \\
u_{x}: g_{v_{x}} g_{u}+g_{u_{x}} f_{u}=\frac{1}{2}\left(g_{v}+f_{u}\right) v_{x}, \quad v_{x}: g_{v_{x}} g_{v}+g_{u_{x}} f_{v}=0, \\
g g_{v}+g_{u} f=0 .
\end{gathered}
$$

Equations (36a) and (37b) gives

$$
f^{2} u_{x}-g^{2} v_{x}=\frac{1}{2}\left(g_{u_{x}}-f_{v_{x}}\right) v_{x}+\frac{1}{2}\left(f_{u_{x}}-g_{v_{x}}\right) u_{x} .
$$

Equations (36b) and (37a) yields

$$
\left(f_{v_{x}}-g_{u_{x}}\right)\left(f_{u_{x}}+g_{v_{x}}\right)=\frac{1}{2}\left(f_{v_{x}}-g_{u_{x}}\right) u_{x}+\frac{1}{2}\left(g_{v_{x}}-f_{u_{x}}\right) v_{x} .
$$

Inserting Equation (38) into Equation (39), one infers that

$$
\left(g_{v_{x}}-f_{u_{x}}\right)\left[\left(f_{u_{x}}+g_{v_{x}}-\frac{1}{2} u_{x}\right)^{2}-\frac{1}{4} v_{x}^{2}\right]=0 .
$$

Detailed calculation shows that the related constraint conditions can be satisfied only if $g_{v_{x}} \neq f_{u_{x}}$. Then we conclude that

$$
\left(f_{u_{x}}+g_{v_{x}}-\frac{1}{2} u_{x}\right)^{2}-\frac{1}{4} v_{x}^{2}=0
$$

which presents

$$
f_{u_{x}}+g_{v_{x}}=\frac{1}{2} u_{x} \pm \frac{1}{2} v_{x}
$$

In what follows, we discuss the solution of Equation (30) in two situations if $f_{u_{x}}+g_{v_{x}}=\frac{1}{2} u_{x}+\frac{1}{2} v_{x}$. For $f_{u_{x}}+g_{v_{x}}=\frac{1}{2} u_{x}-\frac{1}{2} v_{x}$, it can be directly concluded that there is no solution satisfying the constraint conditions.

In particular, we let

$$
\left\{\begin{array}{l}
f_{u_{x}}=\frac{1}{2} v_{x} \\
g_{v_{x}}=\frac{1}{2} u_{x}
\end{array}\right.
$$


whose solution reads

$$
\left\{\begin{array}{l}
f=\frac{1}{2} u_{x} v_{x}+f_{1}\left(v_{x}\right) \\
g=\frac{1}{2} u_{x} v_{x}+g_{1}\left(u_{x}\right) .
\end{array}\right.
$$

Inserting Equation (43) into Equations (38) and (39) gives $g_{1}^{\prime}=f_{1}^{\prime}=0$. That gives $g_{1}=\alpha, f_{1}=\beta$ ( $\alpha$ and $\beta$ are arbitrary constants). It is easily verified that other constraint conditions are automatically satisfied. Hence, one can obtain the Bäcklund transformation of Equation (1) as follows

$$
\left\{\begin{array}{l}
u_{t}=\frac{1}{2} u_{x} v_{x}+\alpha \\
v_{t}=\frac{1}{2} u_{x} v_{x}+\beta
\end{array}\right.
$$

Similarly, we set

$$
\left\{\begin{array}{l}
f_{u_{x}}=\frac{1}{2} u_{x} \\
g_{v_{x}}=\frac{1}{2} v_{x}
\end{array}\right.
$$

which produces $f=\frac{1}{4} u_{x}^{2}+\frac{1}{4} v_{x}^{2}, \quad g=\frac{1}{4} u_{x}^{2}+\frac{1}{4} v_{x}^{2}$. Thus, the second Bäcklund transformation can be expressed as

$$
\left\{\begin{array}{l}
u_{t}=\frac{1}{4} u_{x}^{2}+\frac{1}{4} v_{x}^{2} \\
v_{t}=\frac{1}{4} u_{x}^{2}+\frac{1}{4} v_{x}^{2} .
\end{array}\right.
$$

According to the above derived Bäcklund transformations, we can compute the traveling wave solutions for Equation (44). Firstly, we set $w^{\prime}=0$ and $\alpha=\beta=0$, then $u=v=w$. Thus Equation (44) becomes $w_{t}=\frac{1}{2} w_{x}^{2}$. Letting $\xi=x+c t$, we further get $c w^{\prime}=\frac{1}{2} w^{\prime 2}$, whose solutions read $w=2 c \xi+\gamma$ ( $\gamma$ is arbitrary constant). Furthermore, it is easy to get a traveling wave solution of Equation (26). In fact, setting $w(x, t)=w(\xi), \xi=x+c t$ and substituting into Equation (26), we find $w=\lambda \xi+\mu$ ( $\lambda$ and $\mu$ are arbitrary constants). Because of the arbitrariness of $\gamma, \lambda$ and $\mu$, we find that the solutions of Bäcklund transformations are just that of the original equation Equation (26). Direct computation shows that the solutions that Equation (45) produces are the same as those Equation (44) gives.

\section{Conclusions and Discussion}

In this article, we investigated the Gibbons-Tsarev equation, which arises in reductions of the Benney equation. It turns out the Lie-group analysis method proposed by Olver is a powerful tool for studying the symmetries, invariant solutions, conservation laws of differential equations. Based on obtained symmetry generators, we derive several Lie-group transformations by solving differential equations with the initial condition, and invariant solutions generated by characteristic equations. By supposing that $g(\xi)$ (or $f(\xi)$ ) appearing in the invariant solutions has different rational forms, we may derive fruitful exact solutions. Particularly, we discuss the series solutions of Equation (1) by supposing $g(\xi)=\sum_{n=0}^{\infty} c_{n} \xi^{n}$ in this paper. The new conservation theorem put forward by Ibragimov establishes a connection between symmetries and conservation laws. Hence, on the basis of obtained symmetries, the conservation laws can also be directly constructed via Theorem 1 . Meanwhile, we can also obtain different conservation laws of the original equation by substituting different solutions of the adjoint equation into conserved vectors. In addition, the non-invariant solutions which are different from group-invariant solutions can also be produced from the obtained conservation laws [25]. Here we no longer discuss it. In the last section, we introduced the Bäcklund transformations which are characterized by changing the old solutions into new solutions of the differential equation. Thus, more abundant solutions can be obtained by inserting obtained solutions into the Bäcklund transformations.

Author Contributions: The contributions of the authors are mentioned in this part. Investigation, H.L.; software, H.L.; writing-original draft, H.L.; formal analysis, Y.Z.; project administration, Y.Z.; supervision, Y.Z.; data curation, H.L.; writing-review and editing, H.L. Each author equally contributed to this paper. All authors have read and agreed to the published version of the manuscript. 
Funding: This work was supported by the National Natural Science Foundation of China (grant No.11971475).

Conflicts of Interest: The authors declare no conflict of interest.

\section{References}

1. Olver, P.J. Applications of Lie Groups to Differential Equations; Springer: Berlin/Heidelberg, Germany, 2012.

2. Bluman, G.W.; Cheviakov, A.F.; Anco, S.C. Applications of Symmetry Methods to Partial Differential Equations; Springer: New York, NY, USA, 2010.

3. Ovsiannikov, L.V. Group Analysis of Differential Equations; Academic Press: New York, NY, USA, 1982.

4. Bluman, G.W.; Kumei, S. Symmetries and Differential Equations; Springer: New York, NY, USA, 1989.

5. Krasilshchik, I.S.; Vinogradov, A.M. Nonlocal symmetries and the theory of coverings: An addendum to A. M. vinogradov's "local symmetries and conservation laws". Acta Appl. Math. 1984, 2, 79-96. [CrossRef]

6. Wang, G.W.; Liu, X.Q.; Zhang, Y.Y. Symmetry reduction, exact solutions and conservation laws of a new fifth-order nonlinear integrable equation. Commun. Nonlinear Sci. Numer. Simul. 2013, 18, 2313-2320. [CrossRef]

7. Zhao, Z.; Han, B. Lie symmetry analysis of the Heisenberg equation. Commun. Nonlinear Sci. Numer. Simul. 2017, 45, 220-234. [CrossRef]

8. Zhang, Y.F.; Bai, N.; Guan, H.Y. Some symmetries, similarity solutions and various conservation laws of a type of dispersive water waves. Adv. Differ. Equ. 2019, 2019, 435. [CrossRef]

9. Zhang, Y.; Mei, J.; Zhang, X. Symmetry properties and explicit solutions of some nonlinear differential and fractional equations. Appl. Math. Comput. 2018, 337, 408-418.

10. Ibragimov, N.H.; Avdonina, D.D.E. Nonlinear self-adjointness, conservation laws, and the construction of solutions of partial differential equations using conservation laws. Russ. Math. Surv. 2013, 68, 889. [CrossRef]

11. Lu, H.; Zhang, Y.; Mei, J. Some exact solutions and infinite conservation laws of an extended KdV integrable system. Mod. Phys. Lett. B 2020, doi:10.1142/S0217984920502851. [CrossRef]

12. Gibbons, J.; Tsarev, S.P. Reductions of the Benney equations. Phys. Lett. A 1996, 211, 19-24. [CrossRef]

13. Kaptsov, O.V.; Schmidt, A.V. Linear determining equations for differential constraints. Glasg. Math. J. 2005, 47, 109-120. [CrossRef]

14. Baran, H.; Blaschke, P.; Krasil'Shchik, I.S.; Marvan, M. On symmetries of the Gibbons-Tsarev equation. J. Geom. Phys. 2019, 144, 54-80. [CrossRef]

15. Ibragimov, N.H. Nonlinear self-adjointness in constructing conservation laws. arXiv 2011, arXiv:1109.1728.

16. Ibragimov, N.H. A new conservation theorem. J. Math. Anal. Appl. 2007, 333, 311-328. [CrossRef]

17. Naz, R. Conservation laws for some compacton equations using the multiplier approach. Appl. Math. Lett. 2012, 25, 257-261. [CrossRef]

18. Naz, R.; Mahomed, F.M.; Mason, D.P. Comparison of different approaches to conservation laws for some partial differential equations in fluid mechanics. Appl. Math. Comput. 2008, 205, 212-230.

19. Kara, A.H.; Khalique, C.M. Conservation laws and associated symmetries for some classes of soil water motion equations. Int. J. Nonlinear Mech. 2001, 36, 1041-1045. [CrossRef]

20. Gazizov, R.K.; Ibragimov, N.H.; Lukashchuk, S.Y. Nonlinear self-adjointness, conservation laws and exact solutions of time-fractional Kompaneets equations. Commun. Nonlinear Ence Numer. Simul. 2015, 23, 153-163. [CrossRef]

21. Singla, K.; Gupta, R.K. Conservation laws for certain time fractional nonlinear systems of partial differential equations. Commun. Nonlinear Ence Numer. Simul. 2017, 53, 10-21. [CrossRef]

22. Lukashchuk, S.Y. Conservation laws for time-fractional subdiffusion and diffusion-wave equations. Nonlinear Dyn. 2014, 80, 791-802. [CrossRef]

23. Hongqing, Z.; Yufeng, Z. Bäcklund transformation, nonlinear superposition principle and infinite conservation laws of Benjamin equation. Appl. Math. Mech. 2001, 22, 1017-1021. 
24. Guizhang, T. Bäcklund transformation and conservation laws of the Boussinesq equation. Acta Math. Appl. Sin. 1981, 4, 63-68.

25. Ibragimov, N.H. Conservation laws and non-invariant solutions of anisotropic wave equations with a sourse. Nonlinear Anal. Real World Appl. 2018, 40, 82-94.

(C) 2018 by the authors. Licensee MDPI, Basel, Switzerland. This article is an open access article distributed under the terms and conditions of the Creative Commons Attribution (CC BY) license (http:/ / creativecommons.org/licenses/by/4.0/). 